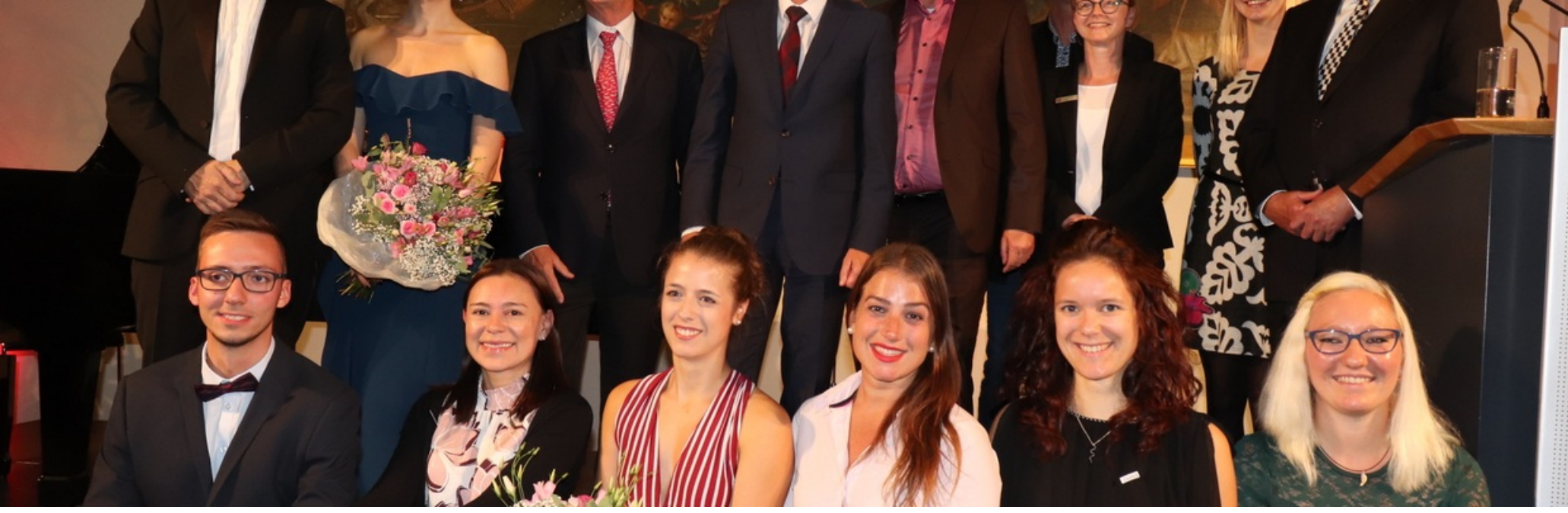

\title{
Art - La mère de toutes les découvertes
}

Authors: Stephan Seiler

Submitted:

4. December 2018

Published:

Volume:

Issue:

Languages:

Keywords:

Categories:

7. December 2018

5

10

French

art, 16.10., Meckelhalle, Strebel, opéra, IASHA e.V. Demetrios News and Views

DOI: $\quad$ 10.17160/josha.5.10.509

\section{Abstract:}

This is a French translation from the original article in German about our yearly award ceremony in Freiburg, Germany. Science meets art! The International Academy of Sciences, Humanities, and Arts organizes a successful concert for the second time. With the motto "Art opens the mind and heart," the deputy chairman of IASHA e. V. Prof. dr. Dr. h.c. mult. Roland Mertelsmann opens the successful symbiosis of music, poetry and scientific talent development. The event was hosted by IASHA e.V. and Marcel Thimm, CEO of Sparkasse Freiburg. The focus is on promoting young scientists and artists through scholarships, prizes and the opportunity to publish in the Journal of Sciences, Humanities, and Arts. 


\section{Art- La mère de toutes les découvertes}

\section{Récital avec la soprano Kim-Lillian Strebel et la poétesse Zazie-Charlotte Pfeiffer}

La science rencontre l'art! L'Académie internationale des sciences, d'humanité et des arts organise un récital de chant pour la deuxième fois. Sous la devise "L'art ouvre l'esprit et le cœur", le viceprésident d'IASHA e. V. Prof. dr. Dr. H. C. mult. Roland Mertelsmann présente une symbiose de la musique, de la poésie et de la promotion scientifique des jeunes talents. IASHA e.V. et Marcel Thimm, PDG de Sparkasse Freiburg organisaient le concert. IASHA e. V. est une organisation à but non lucratif qui a pour objectif de créer et de soutenir les échanges internationaux et interdisciplinaires entre chercheurs, artistes, philosophes et parties intéressées. L'accent est mis sur la promotion des jeunes scientifiques et artistes par le biais de bourses, de récompenses et de la possibilité de publier dans le Journal des sciences humaines et des arts (JOSHA).

\section{Chansons et arias de Kim-Lillian Strebel}

Musicalement, Kim-Lillian Strebel a conçu la soirée. La soprano n'est pas étrangère à Fribourg, puisqu'elle a été membre de l'ensemble du théâtre municipal de 2014 à 2017. Ici, elle a chanté les rôles de Lucette dans Cendrillion de Jules Massenet, le Fiordiligi dans Wolfgang Amadeus dans Così fan tutte et enfin l'Isaure dans la Jérusalem de Giuseppe Verdi. En outre, elle était boursière de l'IASHA e.V. en 2016/2017 et a chanté lors d'un récital à la Sparkasse Freiburg l'année dernière. Kim-Lillian a prouvé qu'elle maîtrisait à la perfection le sujet de

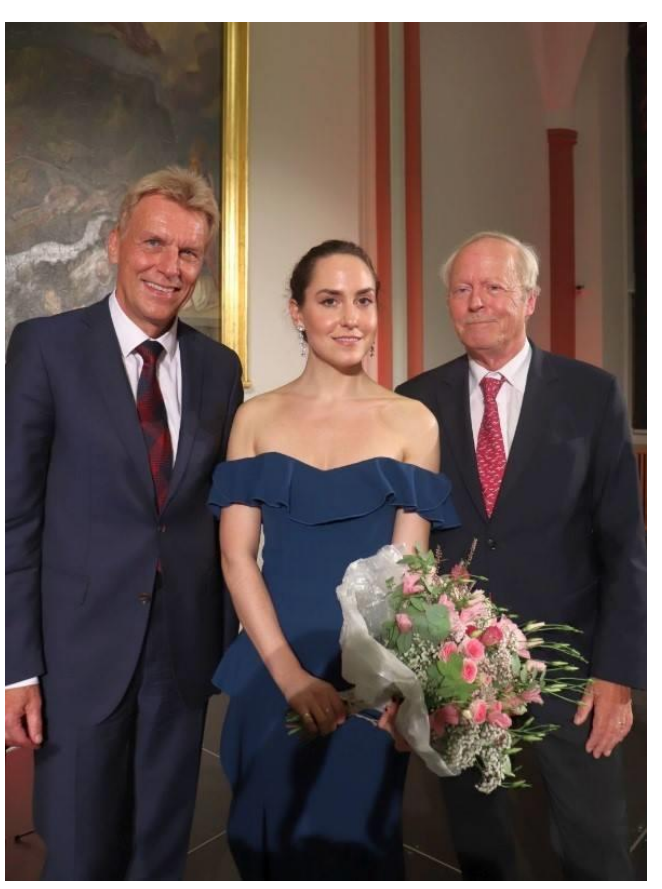
Attribution License http://creativecommons.org/licenses/by/4.0/, which permits unrestricted use, provided the original author and source are credited. 
l'opéra, mais qu'elle jouissait également d'une présence complète en voix et en interprétation dans la section chanson. Ainsi elle a commencé le concert avec une sélection de trois morceaux de la soirée-chanson du compositeur d'opéra Gioachino Rossini. Il a écrit les chansons après avoir terminé sa carrière de compositeur d'opéra et s'est consacré à Paris à la musiqué.

instrumentale, au travail spirituel et à sa passion pour la cuisine. Viennent ensuite deux airs d'opéra du répertoire classique: "Deh vieni, non tardar, o gioia bella" de Nozze di Figaro de Mozart et "Come scolgio" de Così fan tutte. Après la contribution poétique de Zazie-Charlotte Pfeiffer et la remise du prix Demetrios à de jeunes scientifiques, la deuxième partie du concert a été suivie par des arias $\mathrm{du}$ répertoire romantique italo-français. Tout a commencé avec l'aria d'Adelia de l'opéra du même nom de Gaetano Donizetti, dont la première allemande, Kim-Lillian Strebel, a chanté à Hildesheim. Viennent ensuite "O dieu que de bijoux" de Marguerite dans Faust de Charles Gounod et un air de Cendrillon de Jules Massenet, qu'elle a chanté à Fribourg et à partir duquel un DVD est disponible

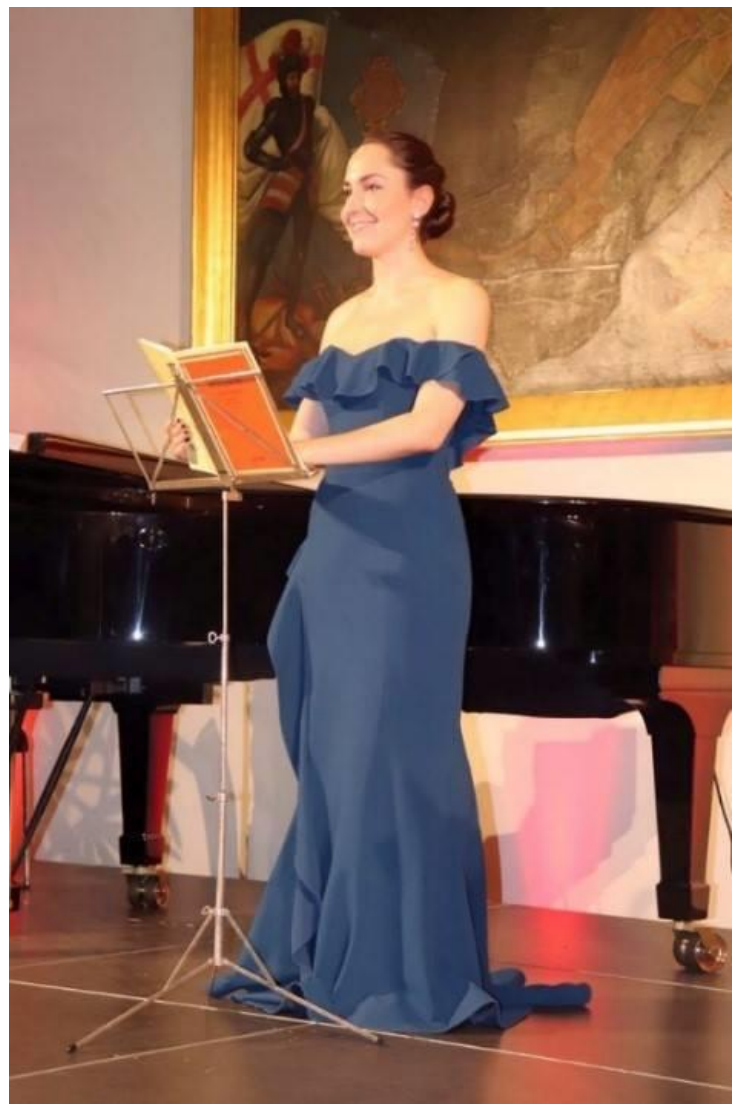
sur le marché. La soirée s'est terminée par un air de Rusalka d'Antonin Dvořák, qu'elle chantera aux États-Unis dans deux ans. Kim-Lillian jouit maintenant d'une renommée internationale et obtient des engagements de l'Allemagne, de l'Italie, de l'Autriche et des États-Unis. Elle possède un large répertoire de chansons, d'oratorio et d'opéra, où elle excelle en tant que soprano lyrique, en particulier dans le sujet classique-romantique. Sa voix commande toutes les couches nécessaires au sujet avec sécurité, couleur et dynamique. Sa représentation des expressions faciales et des gestes va du lyrique au dramatique et n'apparaît à aucun moment. Lors du récital, elle était accompagnée par le pianiste britannique David Cowen. 


\section{Prose de Zazie-Charlotte Pfeiffer}

L'art vit de sa polyvalence, c'est pourquoi Zazie-Charlotte Pfeiffer a conçu la deuxième partie de la soirée avec la présentation de sa nouvelle "Nachts" (« La nuit ») . Le point de départ de l'histoire est une nuit d'été au cours de laquelle la protagoniste ne dort pas et pense à son enfance, à sa relation et à sa vie de jeune personne en général. La même nuit, après une agitation émotionnelle,

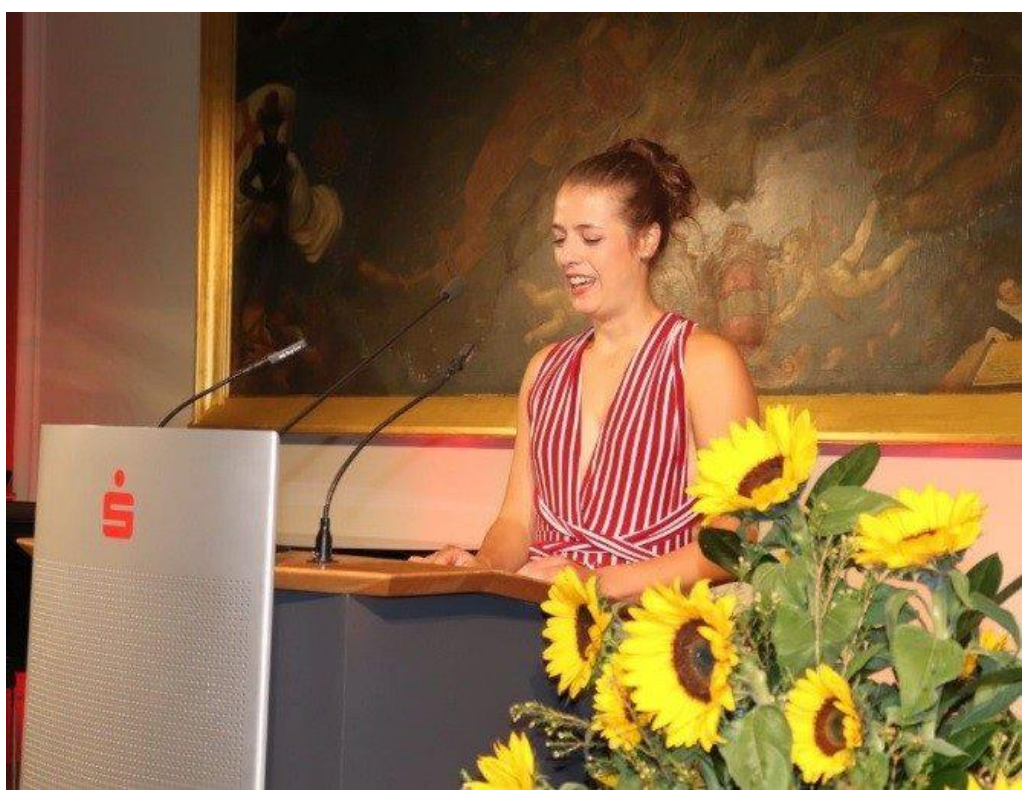
elle vit un moment de beauté et de bonheur. Le sérieux du texte est imprégné d'humour subtil et d'érotisme implicite. Zazie-Charlotte Pfeiffer parvient à créer des images qui sont familières à tous et que tout le monde peut imaginer dans son esprit.

\section{La remise des prix Demetrios}

Après les performances remarquables de Kim-Lillian Strebel et de Zazie-Charlotte Pfeiffer, l'attribution des prix Demetrios à de jeunes scientifiques de l'Académie internationale des sciences, des sciences humaines et des arts fut le point final de la soirée. Le nom du prix est basé sur le philosophe grec Demetrios de Phaleron. Il était l'un des dirigeants de la célèbre bibliothèque d'Alexandrie, où la connaissance du monde antique était réunie. L'IASHA se considère également comme une association dans laquelle le savoir est mis à profit. Ainsi est née l'idée de promouvoir pour la première fois quatre jeunes scientifiques en 2018 au prix de $500 €$. Les lauréats proviennent de différentes disciplines scientifiques: Leah Biebert a reçu un prix pour sa thèse sur "La trilogie Trionfi de Carl Orff" et le rôle joué par Orff dans le Troisième Reich, Elena Riccarda Fellner pour sa thèse sur "La militante, politicienne et intellectuelle iranienne Zahrā Rahnavard et le mouvement iranien des droits des femmes au 20ème siècle ", Nora Marie Leps pour ses travaux scientifiques Attribution License http://creativecommons.org/licenses/by/4.0/, which permits unrestricted use, provided the original author and source are credited. 
sur" La religiosité de la jeunesse chrétienne et musulmane en Allemagne "et Simon Osterkamp pour une" Comparaison des méthodes de caractérisation préclinique d'un produit radiopharmaceutique ". Membre du conseil Prof. Dr. med. Gerhard Steinmann a décerné les prix. Tous les travaux ont également été publiés en ligne dans le Journal des sciences humaines et des arts (www.josha-journal.org).

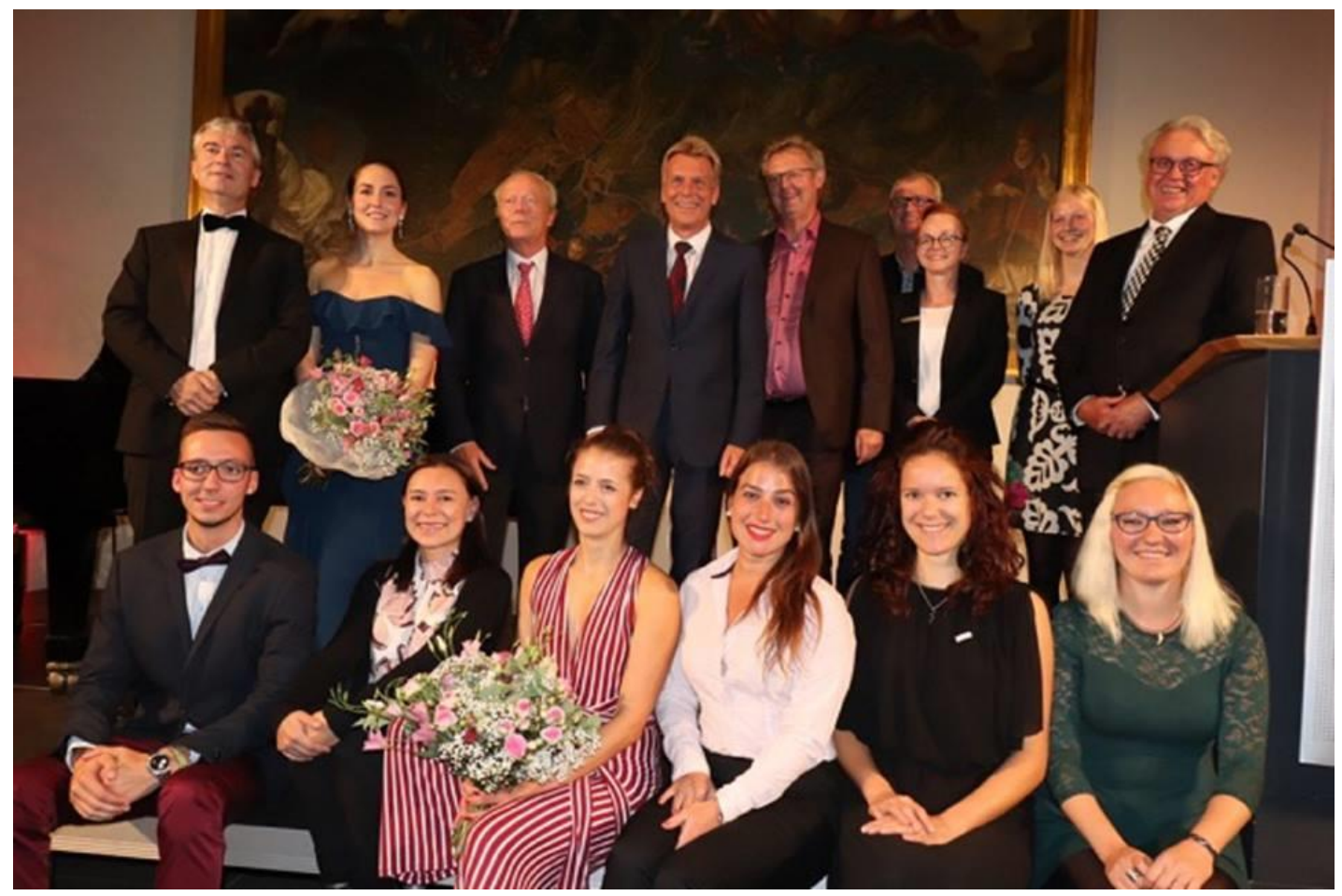

Dr. Stephan Seiler 